\title{
Dinámica de grupo como estrategia pedagógica apoyada en las tecnologías de información y comunicación para fortalecer los valores ${ }^{1}$
}

\section{Group dynamics as a pedagogical strategy supported by information and communication technologies to strengthen values}

DOI: http://dx.doi.org/10.17981/cultedusoc.9.3.2018.0

Artículo de investigación. Fecha de recepción: 15/06/2018. Fecha de aceptación: 27/11/2018

\author{
Gilma Beltrán Ladino²; \\ Yolima Martínez Siado; Luz Puerta Rico; Fernando Jiménez Tapia; \\ Lesfia Portela Villamizar y María Ortiz Dávila ${ }^{3}$ \\ Institución educativa Departamental San José de San Fernando (Colombia) \\ institucionsanjose8645@gmail.com
}

Para citar este artículo:

Beltrán, G., Martínez, Y., Puerta, L., Jiménez, F., Portela, L. y Ortiz, M. (2018). Dinámica de grupo como estrategia pedagógica apoyada en las tecnologías de información y comunicación para fortalecer los valores. Cultura. Educación y Sociedad 9(3), 91-98. DOI: http://dx.doi. org/10.17981/cultedusoc.9.3.2018.0

\section{Resumen}

La dinámica de grupo ha sido una estrategia que junto al uso de las tecnologías de información y comunicación aporta a los procesos de educación, en términos de enseñanza de valores y fortalecimiento de la convivencia escolar. El presente estudio corresponde a fortalecer la sana convivencia y enseñanza de valores mediante las dinámicas de grupo en los estudiantes de primaria del Instituto Educativo Departamental (IED) de San José de San Fernando. La investigación se basa en la Acción Participación (IAP), permite generar conocimientos en forma conjunta para la elaboración de la huerta escolar a los miembros de la comunidad educativa. Los estudiantes consideran necesario proponer y consolidar normas de sana convivencia que sean de conocimiento de todos los estudiantes, docentes y directivos. Se evidencia la necesidad de atender los conflictos a tiempo y motivarlos a su resolución sin violencia, fomentando el impacto positivo que genera la participación de los padres en la educación de sus hijos.

Palabras clave: Sana convivencia, Tics, dinámica de grupo.

\section{Abstract}

Group dynamics has been a strategy that, together with the use of information and communication technologies, contributes to education processes, in terms of teaching values and strengthening school coexistence. The present study corresponds to strengthen the healthy coexistence and teaching of values through group dynamics in elementary students of the Departmental Educational Institute (IED) of San José de San Fernando. The Research is based on the Participation Action (IAP), it allows to generate knowledge jointly for the elaboration of the school garden to the members of the educational community. Students consider it necessary to propose and consolidate standards of healthy coexistence that are known to all students, teachers and managers. There is evidence of the need to address conflicts in time and motivate them to resolve them without violence, fostering the positive impact generated by the participation of parents in the education of their children

Keywords: Healthy coexistence, Tics, group dynamics.

1 Este artículo ha sido derivado del Programa de Fortalecimiento de la Cultura Ciudadana y Democrática CT+I a través de la IEP apoyada en TIC en el Departamento de Magdalena: CICLON

2 Líder del Grupo de Investigación: "Formadores del futuro".

3 Docentes de la institución educativa Departamental San José de San Fernando.

- The author; licensee Universidad de la Costa - CUC

Cultura, Educación y Sociedad vol. 9 no. 3, pp. 91-98. Diciembre, 2018

Barranquilla. ISSN 2389-7724 Online 


\section{Introducción}

En Colombia la Secretaría de Educación de Bogotá, en su compromiso con la convivencia y protección escolar (Dirección Nacional de Estadística, DANE), resalta la necesidad de realizar un diagnóstico sobre la seguridad y convivencia en los colegios identificando como principales problemáticas; el ambiente en el aula (afecciones físicas y Psicológicas), acoso escolar, incidentes de tipo sexual, drogas y armas.

Según el estudio de la Fundación Plan adelantado con 28 , 967 niños de seis regiones, el acoso escolar afecta a 77,5 \% de los estudiantes colombianos.

El (78\% de los niños y $77 \%$ de las niñas). El análisis se adelantó con 28.967 estudiantes de colegios públicos (primaria y bachillerato) de Cauca (en los municipios de Padilla, Suárez, Puerto Tejada y Buenos Aires), Valle del Cauca (en Jamundí), Nariño (Tumaco), Chocó (Quibdó, Tadó, Istmina, Bahía Solano, Río Quito y Condoto), Sucre (Sampués) y Bolívar (Cartagena, Malagana, Arjona, Turbaco, Villanueva y Santa Rosa). En los departamentos del Cauca y Valle del Cauca, participaron del proceso investigativo 20 sedes escolares.

Finalmente, en el departamento del Magdalena, específicamente en San José de San Fernando corregimiento de Santa Ana, La I.E.D San José de San Fernando no es la excepción en cuanto al tema de la Convivencia escolar. Si bien, no existen estadísticas que respalden un estándar de violencia dentro de la institución, es por medio del experiencia y observación por parte de los docentes que se afirma la problemática de convivencia escolar en el aula (Villalobos, Peña, Aguirre y Guerrero, 2017).

De acuerdo con Nail, Muñoz y Gajardo (2013), realizó una investigación principios orientadores de la convivencia en el aula: una estrategia de reflexión colectiva con la Universidad de la Concepción, Concepción, Chile. La investigación llego a las siguientes conclusiones:
Nail, Muñoz y Gajardo (2013) afirma que "Si consideramos que el reglamento de convivencia de los establecimientos educacionales es una herramienta fundamental para la observación de la convivencia escolar, se debe tener presente que una de sus funciones es mejorar el entramado humano al interior de las aulas, normando y posibilitando las relaciones interpersonales entre los sujetos. Por ello, para tener una reflexión significativa de las normas".

Si bien, un manual de convivencia en las Instituciones es considerado de alta importancia debido a la representación con la norma y el orden, es importante destacar que para que se presente un mejoramiento de la convivencia en el aula es necesario realizar una impresión diagnóstica con las problemáticas de cada institución, ya sea desde lo empírico o lo práctico y de esta manera garantizar que las normas que se establezcan en este sean acordes al contexto en el que se va a regir (López de Mesa-Meloa, CarvajalCastillob, Soto-Godoyc y Urrea-Roa, 2013; Olivares, Villón y Iliana, 2011).

Primariamente levantar un diagnóstico con datos empíricos, que permita visibilizar los problemas más importantes y urgentes de la convivencia escolar en los establecimientos.

"Construir un piso común de normas de aula sobre el cual los docentes que lo deseen puedan agregar normas particulares" (Nail, Muñoz y Gajardo, 2013).

En ese orden de ideas se destaca la autonomía que debe tener el docente en su aula, procurando entonces tratar el problema "de raíz" aplicando el caso a caso y de esta manera fortalecer la sana convivencia escolar.

Banz (2008), define las dinámicas de grupo como: "una experiencia social de aprendizaje integral, en la que se conjugan aprendizajes de distinto orden y se propicia la trasferencia de los aprendizajes a la práctica cotidiana. Es una experiencia grupal que dinamiza internamente a cada integrante, promoviendo introspección y reflexión". 
Una de las características más importante de una dinámica grupal es la mirada desde la experiencia, que sin duda alguna representa un mayor grado de entendimiento frente a una temática en particular (De la Cruz, 2015).

\section{Metodología}

Esta investigación utiliza una técnica de evaluación que puede abarcar aspectos cuantitativos y cualitativos. El instrumento utilizado es el cuestionario, que es un procedimiento considerado clásico en las ciencias sociales para obtener y registrar datos. Su versatilidad permite utilizarlo como instrumento de investigación y como instrumento de evaluación de personas, procesos y programas de formación (Montoya, Vanegas y Mejía, 2016).

Se efectuaron visitas a la escuela para observar el comportamiento de los jóvenes durante el descanso y la salida, se realizaron entrevistas al personal docente y directivo de la escuela, con los jóvenes se discutieron dilemas éticos sobre el tema de la amistad, y se aplicó el instrumento referido.

$\mathrm{Su}$ característica singular radica en que, para registrar la información solicitada a los mismos sujetos, ésta tiene lugar de una forma menos profunda e impersonal, que el "cara a cara" de la entrevista. Al mismo tiempo, permite consultar a una población amplia de una manera rápida y económica (García, 2003).

Se utilizó una escala tipo Likert que evaluó diversas dimensiones del abuso entre iguales, así como la actitud de los jóvenes hacia sus maestros y hacia el ambiente escolar. El instrumento se diseñó tomando como base algunas categorías propuestas por el estudio realizado en España, con el fin de permitir la comparación de resultados. En el presente trabajo se analizan el miedo a la escuela, la relación de los alumnos con los maestros, la percepción de los estudiantes tanto como testigos del maltrato entre iguales, como el que han sufrido de manera personal.

Los aspectos que abarca el maltrato son: la exclusión social (me ignoran y me dejan solo; no me dejan participar); el abuso verbal (me ponen apodos que me ofenden y ridiculizan, hablan mal de mí, me insultan), el abuso físico (me pegan) y contra la propiedad (me roban, esconden y rompen las cosas); así como las conductas de intimidación (me amenazan para meterme miedo, me acosan sexualmente, me obligan a hacer algo que no quiero, me amenazan con armas).

TABLA 1

Instrumento: cuestionario

¿Cómo evaluar la convivencia escolar?

La mirada desde los estudiantes

\begin{tabular}{|c|c|c|}
\hline I.E.D SAN J & SE DE SAN F & RNANDO \\
\hline \multicolumn{3}{|l|}{ Curso: } \\
\hline Género: & Masculino () & Femenino ( ) \\
\hline \multirow{6}{*}{\multicolumn{3}{|c|}{$\begin{array}{l}\text { ¿Quiénes viven } \\
\text { en tu casa? } \\
\text { Marca todas } \\
\text { las alternativas } \\
\text { que } \\
\text { correspondan. }\end{array}$}} \\
\hline & & \\
\hline & & \\
\hline & & \\
\hline & & \\
\hline & & \\
\hline Madre & ( ) & \\
\hline Madrastra & ( ) & \\
\hline Padre & ( ) & \\
\hline Padrastro & ( ) & \\
\hline $\begin{array}{l}\text { Hermanos o } \\
\text { hermanas }\end{array}$ & ( ) & \\
\hline $\begin{array}{l}\text { Uno o más de } \\
\text { los abuelos }\end{array}$ & ( ) & \\
\hline $\begin{array}{l}\text { Otros parientes } \\
\text { (tíos, primos, } \\
\text { etc.) }\end{array}$ & ( ) & \\
\hline $\begin{array}{l}\text { Otras personas } \\
\text { que no sean } \\
\text { parientes }\end{array}$ & ( ) & \\
\hline
\end{tabular}




\section{1. ¿Cuán de acuerdo estás con las siguientes afirmaciones?}

\begin{tabular}{lcccc}
\hline & $\begin{array}{c}\text { Muy en } \\
\text { desacuerdo }\end{array}$ & En desacuerdo & $\begin{array}{c}\text { De } \\
\text { acuerdo }\end{array}$ & $\begin{array}{c}\text { Muy de } \\
\text { acuerdo }\end{array}$ \\
\hline $\begin{array}{l}\text { 1.1. Mis padres están pendientes de mi } \\
\text { rendimiento escolar. }\end{array}$ & () & () & () & $($ ) \\
$\begin{array}{l}1.2 \text { Mis padres frecuentemente conversan } \\
\text { con padres de mis compañeros, respecto a } \\
\text { asuntos de mi Colegio. }\end{array}$ & () & & & \\
$\begin{array}{l}1.3 \text { Mis padres se aseguran que yo haga mi } \\
\text { tarea y estudie para los exámenes. }\end{array}$ & () & () & () & () \\
$\begin{array}{l}1.4 \text { Si tengo dificultades con una tarea, pido } \\
\text { ayuda a mis padres. }\end{array}$ & () & () & () & () \\
$\begin{array}{l}1.5 \text { Si mis calificaciones bajan, mis padres se } \\
\text { aseguran que estudie más. }\end{array}$ & () & () & () & () \\
$\begin{array}{l}1.6 \text { Mis padres se preocupan si no mantengo } \\
\text { una buena disciplina en el Colegio }\end{array}$ & () & () & () & () \\
\hline
\end{tabular}

2. Señala tu grado de acuerdo con las siguientes afirmaciones sobre tu Colegio (marca lo que corresponda frente a cada afirmación)

\begin{tabular}{|c|c|c|c|c|c|}
\hline & $\begin{array}{l}\text { Muy en } \\
\text { desacuerdo }\end{array}$ & $\begin{array}{c}\text { En } \\
\text { desacuerdo }\end{array}$ & $\begin{array}{c}\text { De } \\
\text { acuerdo }\end{array}$ & $\begin{array}{l}\text { Muy de } \\
\text { acuerdo }\end{array}$ & $\begin{array}{l}\text { No lo } \\
\text { conozco } \\
\text { o no sé }\end{array}$ \\
\hline $\begin{array}{l}2.1 \text { Conozco las normas de convivencia del } \\
\text { Colegio }\end{array}$ & () & () & () & () & () \\
\hline $\begin{array}{l}2.2 \text { Las normas de convivencia son } \\
\text { respetadas por la mayoría de los estudiantes }\end{array}$ & () & () & () & () & () \\
\hline $\begin{array}{l}2.3 \text { Las normas de convivencia son } \\
\text { respetadas por la mayoría de los profesores }\end{array}$ & () & () & () & () & () \\
\hline $\begin{array}{l}2.4 \text { Los alumnos hemos participado en la } \\
\text { definición de las normas de convivencia del } \\
\text { Colegio }\end{array}$ & () & () & () & () & () \\
\hline $\begin{array}{l}2.5 \text { En casos de faltas a las normas se aplican } \\
\text { las sanciones establecidas en el reglamento } \\
\text { de convivencia }\end{array}$ & () & () & () & () & () \\
\hline $\begin{array}{l}\text { 2.6 Las sanciones se aplican por igual, sin } \\
\text { discriminación a todos los estudiantes }\end{array}$ & () & () & () & ( ) & () \\
\hline $\begin{array}{l}2.7 \text { En mi curso hemos elaborado normas de } \\
\text { convivencia para nuestra aula. }\end{array}$ & () & () & () & () & () \\
\hline $\begin{array}{l}2.8 \text { Cuando hago algo realmente bueno en el } \\
\text { Colegio, mis profesores lo reconocen. }\end{array}$ & () & () & () & ( ) & () \\
\hline $\begin{array}{l}2.9 \mathrm{Si} \text { estoy teniendo problemas personales, } \\
\text { puedo conversar con mis profesores al } \\
\text { respecto. }\end{array}$ & () & () & () & () & () \\
\hline $\begin{array}{l}2.10 \text { Los profesores se ven poco motivados en } \\
\text { su trabajo escolar }\end{array}$ & () & () & () & () & () \\
\hline $\begin{array}{l}\text { 2. } 11 \text { Los profesores de este Colegio motivan } \\
\text { a los estudiantes para que logren su mejor } \\
\text { desempeño. }\end{array}$ & () & () & () & () & () \\
\hline
\end{tabular}


2. 12 Los profesores de este Colegio me hacen sentir que soy capaz de realizar un buen trabajo.

( )

() tratarán de explicármelo de un modo diferente para que logre comprender.

2.14 En general, las clases son entretenidas.

2.15 Los profesores nos preguntan nuestra opinión durante las clases.

( )

( )

( )

( )

el material de su clase con lo que nosotros estamos aprendiendo en otras clases

2.18 Los profesores animan a los estudiantes para participar activamente en las clases.

2.16 Tengo temor de cometer errores en las clases.

(

(

( )

( )

( )

()

() (

( ) ( )

( )

( )

( ) (

( ) ( )

)

( )

( )

( )

()

() $\quad($ ) $\quad() \quad$ ()

( )

( ) ( ) ( )

( )

3. Cuando ocurre un conflicto en el Colegio

¿cuáles son las maneras más comunes de resolverlo?

(Marca solo 2 respuestas)

Dialogando y llegando a acuerdos

Con castigo y sanciones

Sermoneando a quienes se estima son los culpables

Tratando el conflicto entre todos: orientador, coordinador, profesor jefe, alumnos

No dándole importancia y dejándolo pasar

Buscando la ayuda de un mediador.

4. Desde que comenzaron las clases ¿Cuántas veces dentro del Colegio?

\begin{tabular}{|c|c|c|c|c|}
\hline & $\begin{array}{c}0 \\
\text { veces }\end{array}$ & $\begin{array}{c}1 \\
\text { vez }\end{array}$ & $\begin{array}{l}2 \text { o } 3 \\
\text { veces }\end{array}$ & $\begin{array}{l}40 \\
\text { más }\end{array}$ \\
\hline $\begin{array}{l}4.1 \text { ¿has sido víctima del robo o daño intencional de tu propiedad, mp3, } \\
\text { ropa o libros? }\end{array}$ & () & () & () & () \\
\hline 4.2 ¿has sido víctima de rumores o mentiras dañinas sobre ti? & ( ) & ( ) & ( ) & () \\
\hline 4.3 ¿has tenido miedo de que alguien te golpee? & ( ) & ( ) & ( ) & () \\
\hline $\begin{array}{l}4.4 \text { ¿has sido víctima de burlas debido a tu aspecto o por tu manera de } \\
\text { hablar? }\end{array}$ & ( ) & () & $($ ) & () \\
\hline $\begin{array}{l}4.5 \text { ite han empujado, te han hecho a un lado con un empujón, fuiste } \\
\text { golpeado o pateado por alguien que no bromeaba? }\end{array}$ & ( ) & () & $($ ) & () \\
\hline 4.6 ¿has estado en una pelea física? & () & ( ) & () & () \\
\hline 4.7 ¿te han ofrecido, vendido o dado alguna droga ilegal? & $($ ) & ( ) & ( ) & () \\
\hline $\begin{array}{l}4.8 \text { ite han amenazado o lastimado con un arma (una pistola, una navaja o } \\
\text { cuchillo, o una macana o palo, etc.)? }\end{array}$ & ( ) & ( ) & $($ ) & ( ) \\
\hline 4.9 ¿te has sentido acosado sexualmente y has sentido miedo por esa razón? & () & ( ) & ( ) & () \\
\hline 4.10 ¿has dañado intencionalmente la propiedad de la escuela? & () & () & ( ) & () \\
\hline
\end{tabular}

Fuente: adaptación del cuestionario ¿Cómo evaluar la convivencia escolar? La mirada desde los estudiantes (Carrasco, 2009)). 


\section{Resultados}

Por medio de la aplicación de la escala en la Institución Educativa San José de San Fernando y dando respuesta al objetivo general planteado en la investigación se busca indagar sobre la sana convivencia y la enseñanza de valores por medio de dinámicas grupales.

El fortalecimiento de la sana convivencia se logra por medio de la identificación de fortalezas y debilidades que posee la población estudiantil en términos de convivencia, puesto que el empleo del diálogo es de suma importancia a la hora de hacer un proceso de intervención en dificultades de convivencia porque es te debe ser el principal medio para solucionar las dificultades y más dada la posibilidad de llevar a cabo un proceso en un grupo que puede o presenta discrepancias entre sí.

Los estudiantes consideran que es necesario proponer y consolidar normas de sana convivencia que sean de conocimiento de todos los estudiantes, docentes $y$ directivos a fin de crear un ambiente de sana convivencia en la institución educativa.

Los resultados cuantitativos sobre interacciones físicas agresivas y uso de lenguaje fuerte, fueron congruentes con los registros anecdóticos recabados en cada escuela, es decir, en aquella escuela donde se observó. A continuación, se describen los resultados en cinco puntos: el miedo a la escuela, las relaciones con los maestros, el abuso percibido, el abuso sufrido por los encuestados y las diferencias de género.

\section{Discusión}

Luego de la aplicación del cuestionario y las actividades realizadas, se puede inferir que las dinámicas grupales pueden fortalecer la sana convivencia, teniendo en cuenta la necesidad de atender a tiempo los conflictos entre niños y motivarlos a su resolución sin violencia, además de llevar a cabo clases dinámicas introduciendo al juego y motivándolos a la sana convivencia. Es indispensable el trabajo con los padres haciendo énfasis en el buen trato.

El trabajo realizado deja muchas aristas de análisis que quedan pendientes. Lo más importante son los altos y variados patrones de abuso manifiestos. Si bien son preocupantes todas sus formas, vemos que predominan en Colombia los golpes y la coacción ocupan porcentajes muy inferiores a los mostrados en nuestra investigación y cabría reflexionar que en el Magdalena ambas agresiones forman parte de los patrones habituales de crianza y que el comportamiento de los estudiantes es una extensión de lo que viven en otros ambientes.

En el Magdalena, las formas de maltrato con una mayor incidencia son el abuso verbal, se ven de manera natural los chismes, los insultos o los apodos ofensivos, sin pensar que se trata de procesos contrarios a la autoestima y la integridad moral de las personas. Así como señalamos la preocupación sobre el tema, también resultó alentador observar que los motivos más importantes de miedo al ambiente escolar no sean ni los profesores ni los compañeros. Por otro lado, queda como beta de exploración el dato que señala un menor índice de abuso en la I.E.D San José de San Fernando que explícitamente se propuso reducir el uso de las malas palabras entre los niños, niñas y adolescentes de la Institución Educativa.

La violencia escolar en nuestro país, como en algunos otros, alcanza extremos tales como los secuestros exprés entre compañeros, la violación y el asesinato, pero un problema añadido es la violencia de carácter institucional, que es una forma que se añade a las agresiones sufridas. Esto sucede cuando las instituciones, lejos de escuchar y apoyar a las víctimas, niegan el problema e impiden su solución, por 
ejemplo, son bastante conocidos los casos de maltrato que sufren las víctimas cuando siguen un proceso judicial, sobre todo, cuando los implicados en los sucesos han sido los mismos policías. Los careos, frecuentemente, se convierten en una forma de tortura.

Esto también sucede con las autoridades educativas, que cuando finalmente los estudiantes y padres de familia manifiestan sus quejas por abuso, lejos de escuchar e investigar las circunstancias, niegan el problema, protegen a los agresores y exponen a las víctimas. Es el caso, por ejemplo, de quienes recomiendan al agredido y a sus familiares "no decir nada", "por su propio bien”, pues el agresor "está muy bien parado en el sindicato" y puede generarse un ambiente adverso en otras escuelas por el simple hecho de haber denunciado el abuso.

Estos casos de violencia institucional no son privativos del gremio magisterial. Otra forma de agresión sucede también en los hogares donde los padres reprenden y castigan al niño cuando manifiesta que está siendo maltratado en la escuela, por el simple hecho de no haber podido defenderse.

Los casos de maltrato agregado también se hacen evidentes en los medios de comunicación masiva que frecuentemente exponen a las víctimas como si fueran mercancía de ratting. Pero además de la violencia institucional activa, está también la pasiva, es decir, la indiferencia. El hecho de que en los contextos escolares se tolere y se trate como algo "normal", la exime, la legítima y la perpetua. La falta de respeto y los abusos contra las personas en las escuelas se convierten en formas de socialización porque están veladamente permitidas y refrendadas por la propia institución.

Los mismos jóvenes están habituados a sufrir malos tratos y, frecuentemente, prefieren recibir un apodo a ser ignorados por sus compañeros; entonces, como sucedió en la presente investigación, después de señalar que los insultan, les pegan y los amenazan, comentan "nos llevamos bien" o "las relaciones son normales". También, podemos imaginar que, en estas escuelas, los insultos, las burlas, las ofensas, los golpes, a veces, son menos dolorosos que aquellos que los jóvenes han recibido en otros ambientes a los que pertenecen o que observan cotidianamente en el mundo ordinario o en el virtual.

En contraste con el medio, la valoración que hacen de las relaciones sociales en su escuela resulta positiva. Esta aparente "normalidad" y la evaluación positiva de los estudiantes de secundaria sobre las relaciones sociales que se gestan en sus escuelas, lejos de ser una señal de "bienestar subjetivo" probablemente sea un indicador de un proceso de "socialización para la violencia", desde que la propone como algo que hay que tolerar pues forma parte del ser habitual en las escuelas.

Esta percepción distorsionada de la violencia se convierte en parte del medio ambiente, se vuelve "normal", la posibilidad de reconocerla disminuye y, por lo tanto, es introyectada por los sujetos que la viven como algo natural. Esto conduce a acrecentar el problema lejos de disminuirlo, la violencia se reproduce y se vuelve exponencial (Tello, 2005).

Los problemas de violencia escolar no ocurren en un coto de poder aislado del resto de las relaciones sociales que viven los jóvenes, sino que están en perfecta sintonía con el ambiente social, cultural y mediático con el que se relacionan. Las instituciones escolares se muestran ante ello ciegas; lejos de proponer una sociedad mejor a sus alumnos, reproducen automáticamente y fuera de toda conciencia las relaciones de poder que le circundan: las faltas de respeto, los abusos, los malos tratos, los daños a la propiedad ajena, la ley del más fuerte, etcétera 


\section{Referencias}

Banz, C. (2008). Las dinámicas grupales: una técnica de aprendizaje. Chile: Educarchie. Recuperado de http:// ww2.educarchile.cl/UserFiles/P0037/ File/Formacion/Formacion Integral\%2006.pdf

Carrasco, A. (2009). ¿Cómo evaluar la convivencia escolar? La mirada desde los estudiantes?. Revista Iberoamericana de evaluación educativa, 2(1), 258-271.

De la cruz, M. (2015). Dinámicas grupales [Mensaje en un blog]. Recuperado de http://dinamicasgrupalesenf.blogspot. com.co/2015/11/dinamicas-grupales. html

García, T. (2003). El cuestionario como instrumento de investigación/evaluación. Recuperado de http://www.univsantana. com/sociologia/El_Cuestionario.pdf

López de Mesa-Meloa, C., Carvajal-Castillob, C., Soto-Godoyc, M. y Urrea-Roa, P. (2013). Factores asociados a la convivencia escolar en adolescentes. Educ. educ, 16(3). Recuperado de http://www. scielo.org.co/scielo.php?script=sci_artte xt\&pid=S0123-12942013000300001

Montoya, W., Vanegas, A. y Mejía, L. (2016). La evaluación como proceso para la autorregulación de los aprendizajes en el área de ciencias sociales [Tesis de pregado]. Universidad de Antioquia, Medellín. Recuperado de http://ayura.udea.edu.co:8080/jspui/ bitstream/123456789/2343/1/ PB01015_Anacarovanegas_wilmarmontoya.pdf

Nail, O., Muñoz, M. y Gajardo, J. (2013). Principios orientadores de la convivencia en el aula: una estrategia de reflexión colectiva. Educ. Pesqui, 39(2), 367-385.
Núñez Flórez, E., Vergara Ortiz, R. y Bocanegra García, J. (2014). Sistema experto basado en lógica difusa tipo 1 para determinar el grado de riesgo de preeclampsia. INGE CUC, 10(1), 43-50. Recuperado de https://revistascientificas. cuc.edu.co/ingecuc/article/view/341

Olivares, S., Villón, O. y Iliana, C. (2011). Diseño de estrategias mediadoras de conflictos escolares, para mejorar la convivencia educativa en los estudiantes de la Escuela Virgilio Drouet Fuentes, ubicada en el cantón Santa Elena, durante el año 2010-2011. La Libertad. UPSE. Matriz: Facultad de Ciencias de la Educación e Idiomas.

Patiño, A. (2015). Tendencias tecnológicas que influyen en el aumento de la productividad empresarial. INGE CUC, 11(2), 84-96. https://doi.org/10.17981/ingecuc.11.2.2015.09

Quiroz, R. y Saco, S. (2000). Factores asociados al síndrome burnout en médicos y enfermeras del hospital nacional sur este de essalud del Cusco. SITUA - Revista Semestral de la Facultad de Medicina Humana - UNSAAC, 11-22.

Romagnolí, C., Mena, I. y Valdez, A. (2006). ¿Qué son las habilidades socio afectivas y éticas? Recuperado de http://ww2.educarchile.cl/UserFiles/P0037/File/Formacion/Formacion_Integral\%2003.pdf

Tello, N. (2005). Comunidad Segura: modelo de trabajo social para intervenir en el problema de la inseguridad pública. Trabajo Social (revista de la ENTSUNAM).

Villalobos, C., Peña, J., Aguirre, E. y Guerrero, M. (2017). Liderazgo escolar y conflictos socioeducativos. Un estudio exploratorio en liceos públicos chilenos. Calidad en la educación, 47, 81-111. 